\title{
Commentary: Relativity of time in biological systems and stratification of operative risk
}

\author{
Amedeo Anselmi, MD, $\mathrm{PhD},{ }^{\mathrm{a}}$ Xavier Beneux, $\mathrm{MD},{ }^{\mathrm{b}}$ and Nicolas Nesseler, $\mathrm{MD}^{\mathrm{b}}$
}

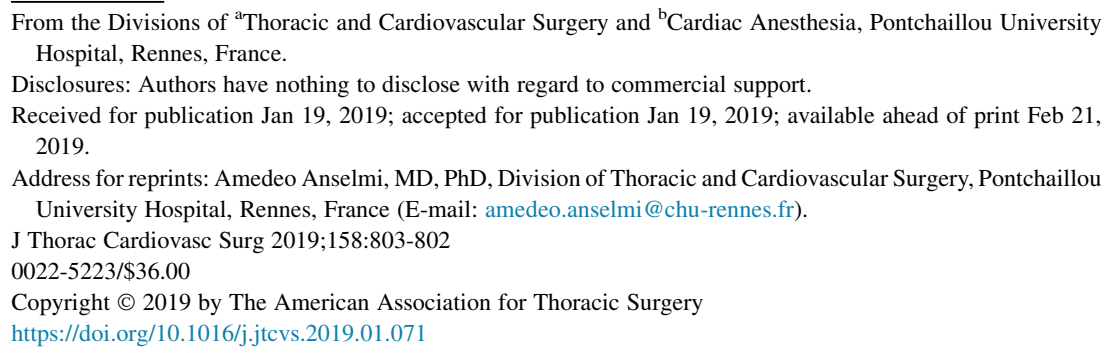

Operative risk assessment represents one of the most crucial issues of modern cardiac surgery in decisionmaking between open and transcatheter techniques, but also in borderline cases in which an assessment of intermediate or high operative risk is needed. Even the best risk score cannot be considered completely reliable when statistically recognized risk factors are not observed; the same can be said about common frailty indexes. Thus, it is our common experience that clinical "eyeballing" from experienced surgeons, in team with experienced cardiac anesthetists, is able to evaluate qualitatively the frailty of a surgical candidate and his/her capability to tolerate and benefit from surgery, and can orient decision-making effectively. Nonetheless, this may be not sufficient when it comes to justify the use of alternative techniques. For instance, in the treatment of valvular heart disease, transcatheter aortic or mitral techniques are associated with a specific profile in periprocedural and postprocedural morbidity, limited follow-up data, and increased immediate economic cost. Therefore, the need for a quantifiable (predictive) marker of senescence, general physiologic capability to tolerate cardiac surgery, and response to this treatment is more current than ever. Similar to what we know in physics, time as a measure of observed changes is relative among individual biological systems. However, because of their great complexity, we lack equations to calculate this gap. The assessment of "biological age" remains qualitative or semiquantitative, and novel biological markers (if effective) have the potential for a great future in the practice.

In this original and appealing investigation, Noly and coworkers ${ }^{1}$ initially hypothesized that plasma levels of the adipokine angiopoietin-like 2 (ANGPTL2), previously associated with chronic proinflammatory status and the burden of cardiovascular disease, could simply decrease after surgery, thus marking the effectiveness of

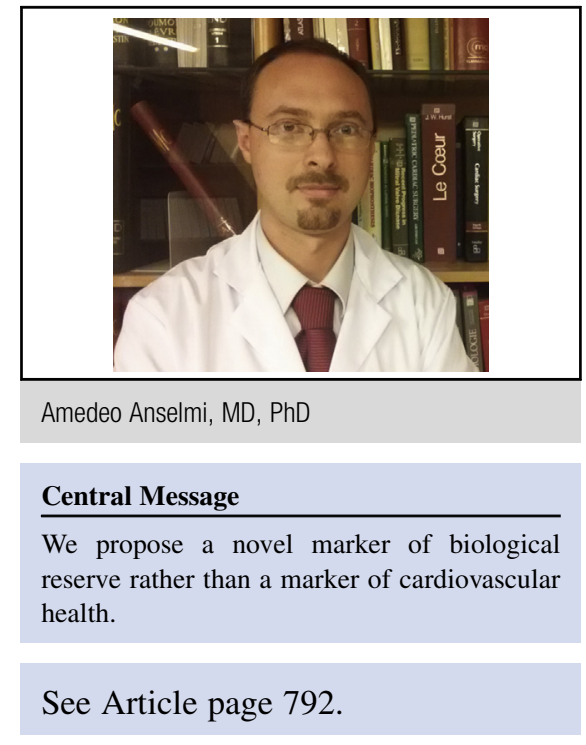

correction of heart disease. One limit to such hypothesis consists of the inclusion in the study of patients with different pathologies (ie, coronary artery disease and aortic valve disease), and the sample size does not allow analysis stratification by the type of baseline pathology. Two alternative profiles in postoperative kinetics of ANGPTL2 were apparent: patients presenting a significant increase or a significant decrease with respect to baseline. Postoperative decrease in ANGPTL2 correlated with a younger age at surgery and lower values of markers of tissue inflammation and arterial senescence. Thus, certain features of ANGPTL2, such as the association with postoperative vasoplegia, concordant with previous findings linking ANGPTL2 with arterial stiffness and late mortality, ${ }^{2}$ or the correlation with known tissue markers of arterial senescence, suggest it should be seen as a marker of "biological" reserve and capability of recovery after treatment of disease rather than a marker of the disease itself. The observation of (paradoxically) greater baseline ANGPTL2 levels in patients with later significant decrease suggests a potential interest as a marker for preoperative use. With this perspective, ANGPTL2 could represent a more useful indicator of biological age as opposed to chronologic age. Nonetheless, major concerns remain for this perspective. Above all, the unexpected observation of preoperative higher levels of ANGPTL2 among younger patients (ie, higher 
ANGPTL2 values could be associated with less senescence in this subgroup) markedly contrasts with the authors' initial hypothesis. This suggests that much is still to be learned about ANGPTL2 before an investigation exploring its potential clinical applicability can be rationally designed. We hope that this dynamic research team will push their future investigations in these directions.

\section{References}

1. Noly P-E, Labbé P, Thorin-Trescases N, Fortier A, Nguyen A, Thorin E, et al. Reduction of plasma angiopoietin-like 2 after cardiac surgery is related to tissue inflammation and senescence status of patients. J Thorac Cardiovasc Surg. 2019; 158:792-802.e5.

2. Desjardins MP, Thorin-Trescases N, Sidibé A, Fortier C, De Serres SA, Larivière R, et al. Levels of angiopoietin-like-2 are positively associated with aortic stiffness and mortality after kidney transplantation. Am J Hypertens. 2017;30:409-16. 